\title{
Hopfield coefficients measured by inverse polariton series
}

\author{
Eiji Tokunaga, ${ }^{1, *}$ A. L. Ivanov, ${ }^{2}$ Selvakumar V. Nair, ${ }^{1, \dagger}$ and Yasuaki Masumoto ${ }^{1,3}$ \\ ${ }^{1}$ ERATO Single Quantum Dot Project, Japan Science and Technology Corporation, 5-9-9 Tokodai, Tsukuba 300-2635, Japan \\ ${ }^{2}$ Department of Physics and Astronomy, Cardiff University, Wales, United Kingdom \\ ${ }^{3}$ Institute of Physics, University of Tsukuba, Tsukuba 305-8571, Japan \\ (Received 28 February 2001; published 30 May 2001)
}

\begin{abstract}
We report observation of the inverse polariton series and a detailed experimental study of the exciton components in polaritons (Hopfield coefficients). Spontaneous emission of excitonic molecules into outgoing polaritons associated with the $i(=1,2,3,4,5)$ th exciton states in bulk $\mathrm{CuCl}$ is detected and analyzed by the bipolariton model. Because the intensities of the emission lines, which form the inverse polariton series, are determined by the exciton components in the final-state polaritons, we are able to measure the Hopfield coefficients for the highly composite outgoing polaritons. Quantum interference in the optical decay of excitonic molecules, due to the multiple exciton components in the polariton and biexciton states, is also demonstrated.
\end{abstract}

DOI: 10.1103/PhysRevB.63.233203

PACS number(s): 71.35.-y, 71.36.+c, 78.55.Hx, 42.65.Yj

The quantum theory of light that resonates with excitons was developed by Hopfield in $1958 .{ }^{1}$ The Hamiltonian for the coupled polarization and electromagnetic fields can readily be diagonalized to generate new eigenstates, polaritons. The polariton eigenstates are characterized by the polariton dispersion and the Hopfield coefficients. The latter describe the relative contributions of the photon and exciton components to the polariton state. The Hopfield coefficients naturally arise in the theoretical descriptions of many phenomena such as polariton scattering, ${ }^{2-6}$ polariton squeezing, ${ }^{7,8}$ and biexciton radiative decay. ${ }^{9,10}$ The polaritons associated with the optically active ground-state excitons in bulk semiconductors have been extensively studied in numerous experiments. ${ }^{11}$ Most of the experiments, however, deal only with the polariton dispersion, while so far there have been no clear measurements of the Hopfield coefficients, for the following reasons. First, in Raman/Brillouin scattering or four-wave mixing experiments, ${ }^{12}$ the Hopfield coefficients combine with the corresponding polariton density of states so that the relevant nonlinear susceptibilities are insensitive to the Hopfield coefficients. ${ }^{3,4,13}$ Secondly, for polaritons in bulk direct-band-gap semiconductors, the Rabi splitting energy is usually larger than the energy band that contributes to the optical signal, i.e., the excitonlike limit of the polariton is adequate in the cases so far studied. ${ }^{1,5}$ By contrast, for microcavity polaritons, ${ }^{6,14}$ the Rabi splitting and the density of states are controlled artificially. Thus the Hopfield coefficients can indeed be experimentally visualized as proposed in Ref. 15, although a rigorous comparison of experiments with theories is absent due to the structural complexities of such systems. In addition to cavity polaritons, a wide variety of artificial polariton systems have been realized in recent years. For example, coupled microcavities and periodic structures of quantum wells and $\operatorname{dots}^{16}$ have been fabricated, leading to a clear demonstration of polariton coupling. These exhibit intrinsically multiple-band polaritons, thus requiring the Hopfield theory to be generalized to multiple oscillators.

In this Brief Report, by studying the optical decay of excitonic molecules (biexcitons) into polaritons associated with the $n s$ exciton, we report a detailed experimental analysis of the Hopfield coefficients in bulk $\mathrm{CuCl}$. In the presence of exciton-exciton interaction, the polariton normal modes are no longer independent oscillators. In the extreme case of biexciton formation the perturbative approach is not valid either for exciton-exciton interaction or for exciton-photon coupling. In this case, in order to treat the above interactions nonperturbatively, one should apply the bipolariton model of an excitonic molecule. ${ }^{17}$ For the resonant dissociation of the biexciton $\mathbf{K}_{m}$ into two outgoing polaritons $\mathbf{k}_{1}^{\text {out }}$ and $\mathbf{k}_{2}^{\text {out }}$, the intensity of the optical signal associated with the lowerbranch polariton $(\mu=1) \mathbf{k}_{1}^{\text {out }}$ is given by

$$
I_{\mu, \nu}\left(\mathbf{k}_{1}^{\text {out }}\right) \propto \rho^{(1,2)}\left|B_{1 s}^{(\mu=1)}\left(k_{1}^{\text {out }}\right) B_{1 s}^{(\nu)}\left(k_{2}^{\text {out }}\right) \epsilon^{m} \int d \mathbf{R} \Psi_{m}(\mathbf{R})\right|^{2},
$$

where $\rho^{(1,2)}$ is the joint density of the polariton states, $\epsilon^{m}$ is the binding energy of the molecule, $B_{i}^{(\nu)}(k)$ is the Hopfield coefficient that characterizes the amplitude of the excitonic polarization associated with the $i$ state in the $\nu$-branch polariton, and $\Psi_{m}(\mathbf{R})$ is the biexciton envelope wave function (WF) as a function of the coordinate $\mathbf{R}$ of the relative motion of the two constituent excitons. Note that the efficiency of the spontaneous decay of biexcitons given by Eq. (1) explicitly depends on the Hopfield coefficients $B_{i}^{(\nu)}$, thus providing us with a unique possibility to measure the Hopfield coefficients.

Single-crystal $\mathrm{CuCl}$ is a prototype material in the physics of polaritons and biexcitons because of the relatively simple valence band structure, the large binding energies of the ground-state exciton and biexciton $\left(\epsilon^{X(1 s)} \simeq 200 \mathrm{meV}\right.$ and $\epsilon^{m} \simeq 32 \mathrm{meV}$ ), and the large polariton Rabi frequency $\left(\hbar \Omega_{X(1 s)} \simeq 224 \mathrm{meV}\right)$. Since the Rabi frequency (the energy scale for the polariton effect) is larger than the biexciton binding energy, the bipolariton model ${ }^{17}$ is necessary to describe the optical decay of molecules. As shown in Fig. 1, below the band gap $(\simeq 3.4 \mathrm{eV})$ there are $Z_{3} 1 s, Z_{1,2} 1 s,{ }^{18}$ and $Z_{3} 2 s, 3 s$, and $4 s$ excitons. ${ }^{19}$ These states give rise to the five polariton branches $\mathrm{LPB}, \mathrm{UPB}$, and $\mathrm{PB}_{2 s, 3 s, 4 s}$, re- 


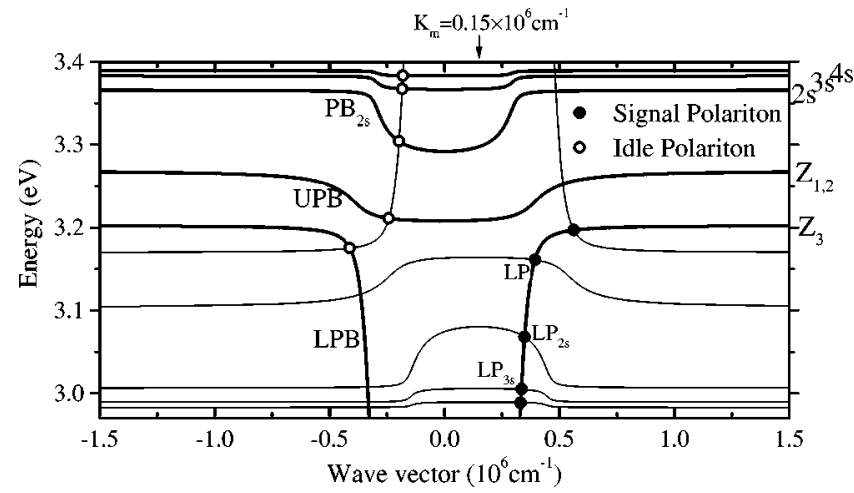

FIG. 1. The multibranch polariton dispersion $\hbar \omega=\hbar \omega_{i}^{\text {pol }}(k)$ associated with $1 s Z_{3}, 1 s Z_{1,2}$, and $(2 s, 3 s, 4 s) Z_{3}$ exciton states in $\mathrm{CuCl}$ (bold lines). The thin curves refer to $\Omega^{m}\left(K_{m}\right)-\omega_{i}^{\text {pol }}\left(K_{m}\right.$ $-k)\left(K_{m}=0.15 \times 10^{6} \mathrm{~cm}^{-1}\right)$, and the points of intersection between the two dispersions correspond to energy-momentum conservation in the optical decay of molecules selectively excited at wave vector $\mathbf{K}_{m}$.

spectively. In Fig. 1 we show a graphical solution of the energy-momentum conservation law in the optical decay of the biexciton state $\mathbf{K}_{m}=\mathbf{k}_{1}^{i n}(\mathrm{LPB})+\mathbf{k}_{2}^{\text {in }}(\mathrm{LPB})$ into multibranch polaritons $\mathbf{k}_{1}^{\text {out }}(\mathrm{LPB})+\mathbf{k}_{2}^{\text {out }}\left(\mathrm{LPB}, \mathrm{UPB}, \mathrm{PB}_{n s}\right) .^{20-22}$ The outgoing polaritons refer to the crossing points between the plotted dispersion curves (in our experiments the signal lower-branch polariton $\mathbf{k}_{1}^{\text {out }}$ is detected in the forward direction, while the conjugated backward-scattered polariton $\mathbf{k}_{2}^{\text {out }}$ is idle). If the biexciton resonant decay into the $\mathrm{PB}_{n s}(n$ $>1$ ) branches is observed, the analysis in the excitonlike limit $^{1,5}\left(B_{1 s}=1\right)$ is no longer adequate because of a wider energy range of observation than the polariton Rabi splitting.

Two synchronized tunable uv picosecond pulses of 2 ps duration, $82 \mathrm{MHz}$ repetition, and $20 \mathrm{~mW}$ average power are delivered through doubling crystals from two TiS lasers electrically synchronized by a Lok-to-Clock circuit (Spectra Physics). The high-repetition-rate picosecond pulses are important for high spectral resolution and for avoiding the higher-order nonlinearities due to reexcitation of biexcitons. ${ }^{23}$ For two-photon generation of biexcitons in $\mathrm{CuCl}$, we change the laser frequencies $\omega_{1}^{i n}$ and $\omega_{2}^{i n}$, keeping the sum of them resonant with the biexciton energy, i.e., $\hbar \omega_{1}^{i n}+\hbar \omega_{2}^{i n}=\hbar \Omega^{m} \simeq 6.372 \mathrm{eV}$. The two circularly polarized laser beams, which are aligned antiparallel and focused into a high-quality $\mathrm{CuCl}$ single crystal at $2 \mathrm{~K}$, induce counterpropagating lower-branch polaritons with wave vectors $\mathbf{k}_{1}^{i n}\left(\omega_{1}^{i n}\right)$ and $\mathbf{k}_{2}^{i n}\left(\omega_{2}^{i n}\right)$. The incoming polaritons selectively generate cold molecules with $\mathbf{K}_{m}=\mathbf{k}_{1}^{i n}+\mathbf{k}_{2}^{\text {in }}$ which spontaneously decay into outgoing polaritons $\mathbf{k}_{1}^{\text {out }}$ and $\mathbf{k}_{2}^{\text {out }}$, mainly before the incoherent scattering processes occur. ${ }^{23}$ Emitted light due to the outgoing polaritons is detected by a $\mathrm{Si}$ charge coupled device camera through a standard fiber bundle and a monochromator.

The emission spectra collected from the transient biexciton states from the band $0 \leqslant\left|\mathbf{K}_{m}\right| \leqslant 0.6 k_{0}$ are plotted in Fig. 2 $\left[\mathbf{K}_{m}=2 \mathbf{k}_{0}\right.$ is the biexciton wave vector of degenerate $\left(\mathbf{k}_{1}^{i n}\right.$ $=\mathbf{k}_{2}^{i n}=\mathbf{k}_{0}$ ) two-polariton excitation, $\left.k_{0} \simeq 0.44 \times 10^{6} \mathrm{~cm}^{-1}\right]$.

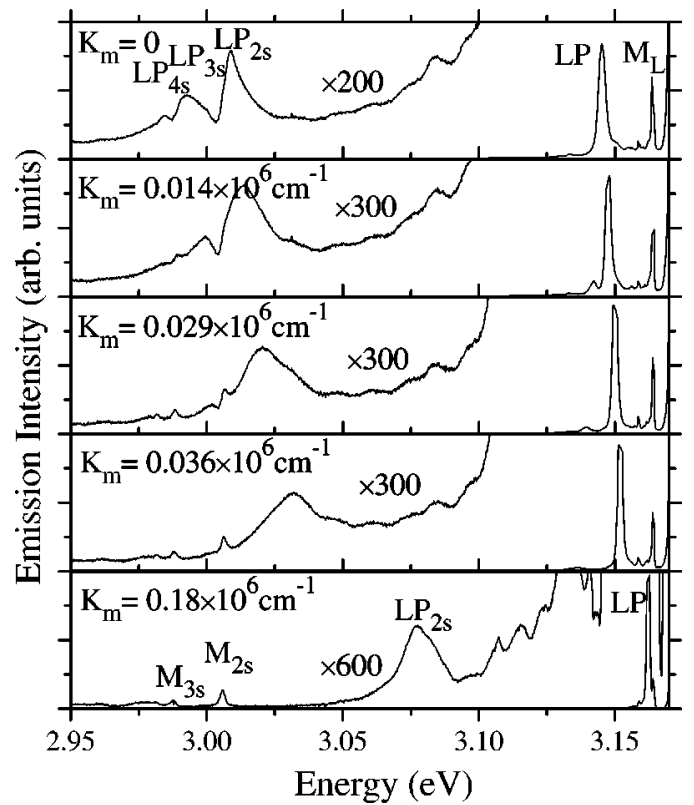

FIG. 2. The inverse polariton series from biexcitons selectively excited at $\mathbf{K}_{m}$ from the band $0 \leqslant K_{m} \leqslant 0.6 k_{0}$. The lines $M_{2 s}, M_{3 s}$, and $M_{L}$ are due to internal surface reflection of one of the incoming polaritons, i.e., due to the change of $\left|\mathbf{K}_{m}\right|$ from $\left|\mathbf{k}_{1}^{i n}+\mathbf{k}_{2}^{i n}\right| \simeq 0$ to $\left| \pm \mathbf{k}_{1}^{i n} \mp \mathbf{k}_{2}^{i n}\right| \simeq 2 k_{0}$. The $M_{L}$ line refers to the optical decay through the longitudinal $1 s Z_{3}$ exciton states. The spectral structure between the $\mathrm{LP}$ and $\mathrm{LP}_{2 s}$ lines is due to emission from bound excitons.

The spontaneous decay $\mathbf{K}_{m} \rightarrow \mathbf{k}_{1}^{\text {out }}(\mathrm{LPB})+\mathbf{k}_{2}^{\text {out }}$ (UPB) gives rise to the doublet of spectral lines called LP and UP. ${ }^{20}$ In Fig. 2, as well as the LP signal, very weak replicas labeled $\mathrm{LP}_{2 s, 3 s, 4 s}$ are also seen on the lower-energy side, due to the channels $\mathbf{K}_{m} \rightarrow \mathbf{k}_{1}^{\text {out }}(\mathrm{LPB})+\mathbf{k}_{2}^{\text {out }}\left(\mathrm{PB}_{2 s, 3 s, 4 s}\right)$. The $\mathrm{LP}_{(n \geqslant 2) s}$ and LP lines form the inverse polariton series. The weak (the intensity ratios $I_{\mathrm{LP}_{n s}} / I_{\mathrm{LP}}$ are of the order of 1/100) but finite transition probabilities into $\mathrm{PB}_{n s}(n>1)$ arise from the $1 s$ exciton components in the outgoing polaritons.

The experimentally determined multibranch polariton dispersion and the component of the $Z_{3} 1 \mathrm{~s}$ exciton in the polariton state are shown in Figs. 3(a) and 3(b) by the filled circles. The solid curves are calculated by a five-oscillator polariton model described below. Figure 3(b) clearly shows how strong is the exciton-photon interaction in $\mathrm{CuCl}$; it redistributes the $Z_{3} 1 s$ exciton component $B_{1 s}$ in the entire energy region over the five-branch polariton dispersion.

The polariton dispersion associated with $N$ exciton states, each characterized by the principal quantum number $n$ and zero orbital angular momentum, is given by

$$
\frac{c^{2} k^{2}}{\varepsilon_{b} \omega^{2}}=1+\sum_{n=1}^{N} \frac{\Omega_{X(n s)}^{2}}{\omega_{X(n s)}^{2}-\omega^{2}},
$$

where $\varepsilon_{b}$ is the background dielectric constant, $\hbar \omega_{X(n s)}$ is the energy of the $X(n s)$ exciton state, and $\Omega_{X(n s)}$ is the corresponding polariton Rabi frequency. The solid curves in Fig. 3 are given by Eq. (2) with $\varepsilon_{b}=4.3, \hbar \omega_{Z_{3}(1 s)}$ $=3.2022 \mathrm{eV}, \hbar \omega_{Z_{1,2}(1 s)}=3.2670 \mathrm{eV}, \hbar \omega_{Z_{3}(2 s)}=3.3665 \mathrm{eV}$, 


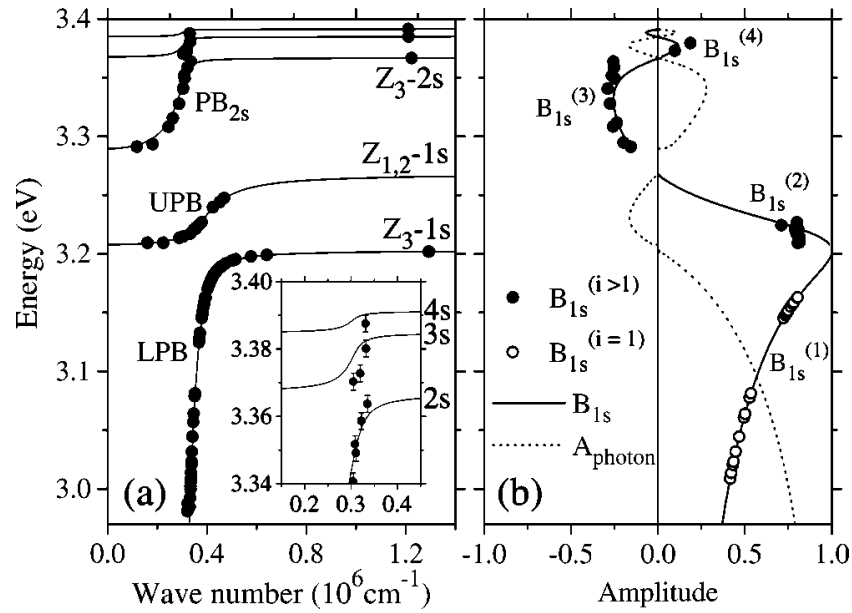

FIG. 3. (a) The multibranch polariton dispersion $\hbar \omega$ $=\hbar \omega_{i}^{\text {pol }}(k)$ measured for bulk $\mathrm{CuCl}$ at $T=2 \mathrm{~K}$. (b) The generalized Hopfield coefficients $A^{(\nu)}=A^{(\nu)}(\hbar \omega)$ (photon component) and $B_{1 s}^{(\nu)}=B_{1 s}^{(\nu)}(\hbar \omega)\left(Z_{3} 1 s\right.$ exciton component), deduced from the experimental data (filled circles) and calculated by Eqs. (3) and (4) (dotted and solid curves, respectively).

$\hbar \omega_{Z_{3}(3 s)}=3.3846 \mathrm{eV}, \quad \hbar \omega_{Z_{3}(4 s)}=3.3911 \quad \mathrm{eV}, \quad \hbar \Omega_{Z_{3}(n s)}$ $=223.6 / n^{3 / 2} \mathrm{meV}$ for $n=1,2,3,4$, and $\hbar \Omega_{Z_{1,2}(1 s)}=371 \mathrm{meV}$.

The Hopfield coefficients for multibranch polaritons are obtained by generalization of the Hopfield theory. ${ }^{1,3}$ At each spectral point $\left[\mathbf{k}, \omega_{\nu}^{p o l}(\mathbf{k})\right]$ the $\nu$-branch polariton consists of the photon and $N$ exciton components specified by the generalized Hopfield coefficients

$$
\begin{gathered}
A^{(\nu)}=-\left(\frac{c k}{\varepsilon_{b}^{1 / 2} \omega_{\nu}^{p o l}}\right)^{1 / 2} \frac{1}{D_{\nu}}, \\
B_{n}^{(\nu)}=i\left(\frac{\Omega_{X(n s)}}{\omega_{X(n s)}}\right) \frac{\left(x_{n}^{\nu}\right)^{1 / 2}}{1-\left(x_{n}^{\nu}\right)^{2}} \frac{1}{D_{\nu}},
\end{gathered}
$$

where $\quad x_{n}^{\nu}=\omega_{\nu}^{p o l} / \omega_{X(n s)} \quad$ and $\quad D_{\nu}=\left\{1+\sum_{n=1}^{N}\left(\Omega_{X(n s)} /\right.\right.$ $\left.\left.\omega_{X(n s)}\right)^{2}\left[1-\left(x_{n}^{\nu}\right)^{2}\right]^{-2}\right\}^{1 / 2}$. The relative contributions of the photon and exciton components to the $\nu$ th-branch polariton $\mathbf{k}$ are given by $\left|A^{(\nu)}(k)\right|^{2}$ and $\left|B_{n=1, \ldots, N}^{(\nu)}(k)\right|^{2}$, respectively, so that $\left|A^{(\nu)}\right|^{2}+\sum_{n=1}^{N}\left|B_{n}^{(\nu)}\right|^{2} \simeq 1$ except for $k \simeq 0$. Here, damping constants $\gamma_{n s}$ for $n s$ excitons $(n>1)$, which are estimated to be a few meV, ${ }^{10}$ are neglected because $\Omega_{X(n s)}$ is much larger than $\gamma_{n s} .{ }^{24}$

For the evaluation of the experimental points in Fig. 3(a), using the energies of the LP lines and known values for the biexciton energy $6.372 \mathrm{eV}$ and $Z_{3} 1 s$ exciton parameters, we adjust $\varepsilon_{b}$ and the $Z_{1,2} 1 \mathrm{~s}$ exciton parameters to obtain the best fit to the data. For Fig. 3(b), we assume that $B_{1 s}^{(1)}\left(k_{1}^{\text {out }}\right)$ satisfies Eq. (4) (open circles) and $B_{1 s}^{(\nu)}\left(k_{2}^{\text {out }}\right)$ is determined by $B_{1 s}^{(\nu)}\left(k_{2}^{\text {out }}\right)=C \sqrt{\left(I_{\mathrm{LP}_{n s}} / I_{M_{L}}\right) /\left(\rho^{(1,2)} T\right)} / B_{1 s}^{(1)}\left(k_{1}^{\text {out }}\right)$, where $T$ is the sample transmissivity and the constant $C$ is so taken that one point of $B_{1 s}\left(k_{2}^{\text {out }}\right)$ falls on the theoretical curve. Here, $I_{\mathrm{LP}_{n s}} / I_{M_{L}}$ is the intensity normalized by the biexciton density, ${ }^{13,25}$ which is estimated from the relative intensity of the $\mathrm{LP}_{n s}$ line with respect to the $M_{L}$ line for each spectrum plotted in Fig. 2, and the sign of the experimental $B_{1 s}^{(\nu)}$ is assumed to follow the theory. The theoretical curves calculated by Eqs. (2)-(4) agree well with the experimental data.

One can still see residual differences between the experimental and theoretical results plotted in the inset of Fig. 3(a). For the theoretical curve, the $n s$ exciton oscillator strength is assumed to follow the $1 / n^{3}$ law, while the experimental points around $\mathrm{PB}_{3 s}$ and $\mathrm{PB}_{4 s}$ deviate from the theoretical curve. This demonstrates violation of the $1 / n^{3}$ law for excitons in $\mathrm{CuCl}$. The latter conclusion is consistent with the deviation of the binding energies from the $1 / n^{2}$ law. ${ }^{19}$ In order to fit the dispersion, the oscillator strengths are found to be less than one-half of those estimated by the $1 / n^{3}$ law for $n=2,3,4$. For $B_{1 s}^{(3,4)}$ plotted in Fig. 3(b), there is also some discrepancy between the experimental and theoretical results in the spectral region close to the $(2,3) s$ exciton resonances. This comes mainly from the approximations in Eq. (1), where the contributions from the $n s(n>1)$ exciton states to the molecule WF, $C_{1, n}$, are neglected. Thus the $n s$ $(n>1)$ exciton component $B_{n}$ does not appear in Eq. (1). The above contributions give rise to the recently observed inverse exciton $M$ emission series, ${ }^{10}$ which occurs in the biexciton optical decay that leaves behind almost pure $n s$ excitons.

A more precise expression for the outgoing intensity $I_{1, \nu}$ based on the bipolariton model reads ${ }^{10}$

$$
I_{1, \nu}\left(\mathbf{k}_{1}^{\text {out }}\right) \propto \rho^{(1,2)}\left|\sum_{i, j} B_{i}^{(1)}\left(k_{1}^{\text {out }}\right) B_{j}^{(\nu)}\left(k_{2}^{\text {out }}\right) W_{i, j} C_{i, j}\right|^{2},
$$

where $C_{i, j}=\int d \mathbf{R} d \mathbf{r}_{1} d \mathbf{r}_{2} \Gamma_{m}^{*}\left(\mathbf{R}, \mathbf{r}_{1}, \mathbf{r}_{2}\right) \phi_{i}\left(\mathbf{r}_{1}\right) \phi_{j}\left(\mathbf{r}_{2}\right), \quad W_{i, j}=$ $-\epsilon^{m}-\epsilon^{x}\left(2-1 / i^{2}-1 / j^{2}\right)$, and $\phi_{n}$ is the $n s$ exciton envelope WF. Equation (1) is obtained as a special case of Eq. (5) by using the simplified molecule WF, $\Gamma_{m}\left(\mathbf{R}, \mathbf{r}_{1}, \mathbf{r}_{2}\right)$ $=\Psi_{m}(\mathbf{R}) \phi_{1}\left(\mathbf{r}_{1}\right) \phi_{1}\left(\mathbf{r}_{2}\right)$. At $\mathbf{K}_{m}=2 \mathbf{k}_{0}$ the inverse polariton series reduces to the inverse exciton $M$ series. The intensity of the $M_{n s}$ lines is determined by the decay path $(i=1, j$ $=n), I_{M_{n s}} \propto\left|B_{1 s}^{(\mu=1)}\left(k_{1}^{\text {out }}\right) B_{n s}^{(\nu=n+1)}\left(k_{2}^{\text {out }}\right) W_{1, n} C_{1, n}\right|^{2}$. By analyzing the $M$ series, we have already found the absolute values $\left|C_{2} / C_{1}\right|,\left|C_{3} / C_{1}\right|$, and $\left|C_{4} / C_{1}\right|$, where $C_{n} \equiv C_{1, n} \cdot{ }^{10} \mathrm{In}$ the five-oscillator model given by Eqs. (2)-(4), the outgoing polaritons $\mathbf{k}_{1,2}^{\text {out }}$ generally consist of a photon and five exciton $\left(1 s, 2 s, 3 s, 4 s Z_{3}\right.$ and $\left.1 s Z_{1,2}\right)$ components. Hence, the $\left|\mathbf{K}_{m}\right|$-dependent intensity of the $\mathrm{LP}_{n s}$ lines $I_{\mathrm{LP}}$ $=I_{\mathrm{LP}_{n s}}\left(\left|\mathbf{K}_{m}\right|\right)$ allows us to determine unambiguously the signs of $C_{n}$ as $C_{1}=1, C_{2}=-0.0125, C_{3}=-0.0066$, and $C_{4}=-0.0042$. This is done by examining quantum interference, constructive or destructive, between the paths $(i=1, j$ $=1)$ and $(i=1, j=n)$ with the use of Eq. (4) for $B_{n s}$. The LP line is predominantly determined by the path $(1,1)$. The intensity of the $\mathrm{LP}_{2 s}$ line, on the other hand, depends not only on the path $(1,1)$ but also on the path $(1,2)$ as the idle polariton approaches the $2 s$ exciton, i.e.,

$$
\begin{aligned}
I_{\mathrm{LP}_{2 s}} \propto & \mid B_{1 s}^{(1)}\left(k_{1}^{\text {out }}\right) B_{1 s}^{(3)}\left(k_{2}^{\text {out }}\right) W_{1,1} C_{1,1} \\
& +\left.B_{1 s}^{(1)}\left(k_{1}^{\text {out }}\right) B_{2 s}^{(3)}\left(k_{2}^{\text {out }}\right) W_{1,2} C_{1,2}\right|^{2} .
\end{aligned}
$$


The two spontaneous decay paths interfere constructively to give nearly equal contributions to the $\mathrm{LP}_{2 s}$ intensity at $K_{m}$ $=0$, which should otherwise be much less than observed. Further, the $\mathrm{LP}_{3 \text { s }}$ line is determined by three paths $(1,1)$, $(1,2)$, and $(1,3)$, i.e.,

$$
\begin{aligned}
I_{\mathrm{LP}_{3 s}} \propto & \mid B_{1 s}^{(1)}\left(k_{1}^{\text {out }}\right) B_{1 s}^{(4)}\left(k_{2}^{\text {out }}\right) W_{1,1} C_{1,1} \\
& +B_{1 s}^{(1)}\left(k_{1}^{\text {out }}\right) B_{2 s}^{(4)}\left(k_{2}^{\text {out }}\right) W_{1,2} C_{1,2} \\
& +\left.B_{1 s}^{(1)}\left(k_{1}^{\text {out }}\right) B_{3 s}^{(4)}\left(k_{2}^{\text {out }}\right) W_{1,3} C_{1,3}\right|^{2} .
\end{aligned}
$$

For $\left|\mathbf{K}_{m}\right|=0$ the first and third paths are dominant and interfere constructively. For $K_{m}>0.05 \times 10^{6} \mathrm{~cm}^{-1}$, the $\mathrm{LP}_{3 s}$ line almost disappear (see Fig. 2) due to destructive interference of the second path with the first and third ones. The $K_{m}$ dependence of the $\mathrm{LP}_{4 s}$ line is explained similarly.

In conclusion, we have measured the Hopfield coefficients for highly composite polaritons in $\mathrm{CuCl}$ and verified the Hopfield theory generalized to multioscillator polaritons.

We appreciate valuable discussions with M. KuwataGonokami and N. Nagasawa, and thank K. Kurihara for sample purification. A.L.I. acknowledges support by EPSRC.
*Present address: Department of Physics, University of Tokyo, Hongo 7-3-1, Bunkyo, Tokyo 113-0033, Japan.

†Present address: National Renewable Energy Laboratory, Golden, Colorado 80401.

${ }^{1}$ J.J. Hopfield, Phys. Rev. 112, 1555 (1958); 182, 945 (1969).

${ }^{2}$ C. Weisbuch and R.G. Ulbrich, in Light Scattering in Solids III, Vol. 51 of Topics in Applied Physics, edited by M. Cardona and G. Güntherodt (Springer, Berlin, 1982), p. 207.

${ }^{3}$ D.L. Mills and E. Burstein, Phys. Rev. 188, 1465 (1969).

${ }^{4}$ B. Bendow, Phys. Rev. B 2, 5051 (1970); in Electronic Structure of Noble Metals and Polariton Mediated Light Scattering, Vol. 82 of Springer Tracts in Modern Physics (Springer, Berlin, 1978), p. 69.

${ }^{5}$ M. Matsushita, J. Wicksted, and H.Z. Cummins, Phys. Rev. B 29, 3362 (1984).

${ }^{6}$ F. Tassone, C. Piermarocchi, V. Savona, A. Quattropani, and P. Schwendimann, Phys. Rev. B 56, 7554 (1997); F. Tassone and Y. Yamamoto, ibid. 59, 10830 (1999).

${ }^{7}$ A. Quattropani, L.C. Andreani, and F. Bassani, Nuovo Cimento D 7, 55 (1986); P. Schwendimann, A. Quattropani, and Z. Hradil, ibid. 15, 1421 (1993).

${ }^{8}$ M. Artoni and J.L. Birman, Phys. Rev. B 44, 3736 (1991).

${ }^{9}$ F. Henneberger and J. Voigt, Phys. Status Solidi B 76, 313 (1976)

${ }^{10}$ E. Tokunaga, A.L. Ivanov, S.V. Nair, and Y. Masumoto, Phys. Rev. B 59, R7837 (1999).

${ }^{11}$ E.S. Koteles, in Excitons, edited by E.I. Rashba and M.D. Sturge (North-Holland, Amsterdam, 1982), p. 83.

${ }^{12}$ A. Maruani and D.S. Chemla, Phys. Rev. B 23, 841 (1981).

${ }^{13}$ A.L. Ivanov, M. Hasuo, N. Nagasawa, and H. Haug, Phys. Rev. B 52, 11017 (1995)
${ }^{14}$ C. Weisbuch, M. Nishioka, A. Ishizawa, and Y. Arakawa, Phys. Rev. Lett. 69, 3314 (1992); for a review, see G. Khitrova, H.M. Gibbs, F. Jahnke, M. Kira, and S.W. Koch, Rev. Mod. Phys. 71, 1591 (1999).

${ }^{15}$ W.R. Tribe, D. Baxter, M.S. Skolnick, D.J. Mowbray, T.A. Fisher, and J.S. Roberts, Phys. Rev. B 56, 12429 (1997); A. Fainstein, B. Jusserand, and R. André, ibid. 57, 9439 (1998).

${ }^{16}$ A. Armitage, M.S. Skolnick, V.N. Astratov, D.M. Whittaker, G. Panzarini, L.C. Andreani, T.A. Fisher, J.S. Roberts, A.V. Kavokin, M.A. Kaliteevski, and M.R. Vladimirova, Phys. Rev. B 57, 14877 (1998); T. Gutbrod, M. Bayer, A. Forchel, J.P. Reithmaier, T.L. Reinecke, S. Rudin, and P.A. Knipp, ibid. 57, 9950 (1998); M. Hübner, J.P. Prineas, C. Ell, P. Brick, E.S. Lee, G. Khitrova, H.M. Gibbs, and S.W. Koch, Phys. Rev. Lett. 83, 2841 (1999).

${ }^{17}$ A.L. Ivanov, H. Haug, and L.V. Keldysh, Phys. Rep. 296, 237 (1998).

${ }^{18}$ D. Fröhlich, P. Köhler, W. Nieswand, and E. Mohler, Phys. Status Solidi B 167, 147 (1991).

${ }^{19}$ K. Saito, M. Hasuo, T. Hatano, and N. Nagasawa, Solid State Commun. 94, 33 (1995).

${ }^{20}$ T. Itoh and T. Suzuki, J. Phys. Soc. Jpn. 45, 1939 (1978).

${ }^{21}$ T. Mita, K. Sôtome, and M. Ueta, Solid State Commun. 33, 1135 (1980).

${ }^{22}$ B. Hönerlage, R. Lévy, J.B. Grun, C. Klingshirn, and K. Bohnert, Phys. Rep. 124, 161 (1985).

${ }^{23}$ H. Akiyama, T. Kuga, M. Matsuoka, and M. Kuwata-Gonokami, Phys. Rev. B 42, 5621 (1990).

${ }^{24}$ T. Skettrup, Phys. Rev. B 24, 884 (1981).

${ }^{25}$ K. Kurihara, E. Tokunaga, M. Baba, and M. Matsuoka, Phys. Rev. B 52, 8179 (1995). 